\title{
Addressing and proposing solutions for unmet clinical needs in the management of myeloproliferative neoplasm-associated thrombosis: A consensus-based position paper
}

\author{
Tiziano Barbui ${ }^{1}$, Valerio De Stefano $\mathbb{E}^{2,3}$, Anna Falanga $\mathbb{E}^{4,5}$, Guido Finazzi ${ }^{6}$, Ida Martinelli ${ }^{7}$, Francesco Rodeghiero ${ }^{8}$, \\ Alessandro M. Vannucchi ${ }^{9}$ and Giovanni Barosi ${ }^{10}$
}

\begin{abstract}
This article presents the results of a group discussion among an ad hoc constituted Panel of experts aimed at highlighting unmet clinical needs (UCNs) in the management of thrombotic risk and thrombotic events associated with Philadelphia-negative myeloproliferative neoplasms (Ph-neg MPNs). With the Delphi technique, the challenges in Ph-neg MPN-associated thrombosis were selected. The most clinically relevant UCNs resulted in: (1) providing evidence of the benefits and risks of direct oral anticoagulants, (2) providing evidence of the benefits and risks of cytoreduction in patients with splanchnic vein thrombosis without hypercythemia, (3) improving knowledge of the role of the mutated endothelium in the pathogenesis of thrombosis, (4) improving aspirin dosing regimens in essential thrombocythemia, (5) improving antithrombotic management of Ph-neg MPN-associated pregnancy, (6) providing evidence for the optimal duration of anticoagulation for prophylaxis of recurrent VTE, (7) improving knowledge of the association between somatic gene mutations and risk factors for thrombosis, and (8) improving the grading system of thrombosis risk in polycythemia vera. For each of these issues, proposals for advancement in research and clinical practice were addressed. Hopefully, this comprehensive overview will serve to inform the design and implementation of new studies in the field.
\end{abstract}

\section{Introduction}

Classical Philadelphia-negative myeloproliferative neoplasms (Ph-neg MPNs) include polycythemia vera (PV), essential thrombocythemia (ET), and myelofibrosis (MF). The discovery that driver somatic mutations (primarily $J A K 2 \mathrm{~V} 617 \mathrm{~F})$ and additional subclonal mutations contribute to the pathogenesis of $\mathrm{Ph}$ neg-MPNs ${ }^{1}$ has opened a new era in both the biological understanding of these disorders and the associated clinical practice. In addition,

\footnotetext{
Correspondence: Tiziano Barbui (tbarbui@fondazionefrom.it)

${ }^{1}$ FROM Research Foundation, Papa Giovanni XXIII Hospital, Bergamo, Italy

${ }^{2}$ Fondazione Policlinico Universitario A. Gemelli IRCCS, Roma, Italy

Full list of author information is available at the end of the article.
}

new advances in the knowledge of molecular pathogenesis have also improved our understanding of the pathogenesis of MPN-associated arterial and venous major thrombosis, and new data on the complex interplay among blood cells, endothelium and coagulation system are now available ${ }^{2}$. Therefore, major advances have been attained in terms of prognostic classification and, interestingly, new therapeutic hypotheses about thrombotic risk and events have been raised ${ }^{3-5}$.

Despite this increase in knowledge, arterial thrombosis and venous thromboembolism (VTE) remain the principal causes of morbidity and mortality in patients with $\mathrm{Ph}$ neg $\mathrm{MPNs}^{6,7}$. Thrombosis can occur from the very early 
stages of the disease and complicates the course of the follow-up ${ }^{6}$ with an incidence of either arterial thrombosis or VTE approximately fivefold to tenfold higher than in the general population ${ }^{6}$. This increase is mostly because new concepts for thrombosis management were translated from a limited number of interventional clinical studies ${ }^{8}$.

In this review, we attempt to define the unmet clinical needs (UCNs) in Ph-neg MPN-associated thrombosis, which relate principally to an improvement in risk classification, trial design, and structured care delivery. For this aim, a group of experts was convened to participate in a special consensus project.

\section{Methods}

A Panel of Italian experts (hereafter referred to as the Panel) was selected based on the individual expertise in research and clinical practice with Ph-neg MPNs. During an initial meeting on June 2018, the outline of the project was discussed, and the topics that form the structure of the present document were decided. The key UCNs were selected through a series of questionnaires according to the Delphi technique ${ }^{9}$. Afterward, each panelist drafted statements addressing one identified $\mathrm{UCN}$, and the remaining panelists scored their agreement with those statements and provided suggestions for modifications.
Finally, the Panel convened for a consensus conference held in Milano, Italy, in October 2018. At this conference, the final proposals were given using the nominal group technique ${ }^{9}$, by which participants including European experts and members of the European Leukemia Net (ELN), were first asked to comment on their disagreements with the proposed issues in round-robin fashion and then to vote for a final statement. The resulting general discussion provided remarkable input to the present report.

\section{Results}

Although numerous UCNs in Ph-neg MPNs were issued by the Panel (Table 1), this review focuses on some of the major outstanding challenges voted as the most relevant and urgent by the panelists.

\section{UCN1: providing evidence of the benefits and risks of direct oral anticoagulants in Ph-neg MPNs}

In the general population, the direct oral anticoagulants (DOACs) dabigatran, rivaroxaban, apixaban, and edoxaban are approved for treatment of $\mathrm{VTE}^{10}$, perioperative thromboprohylaxis, and ischemic stroke prevention in atrial fibrillation $(\mathrm{AF})^{10,11}$. In these settings, DOACs are as effective as warfarin and have a lower rate of intracranial hemorrhage.

\section{Table 1 Candidate unmet clinical needs (UCNs)}

\begin{tabular}{|c|c|}
\hline No. & Candidate UCNs \\
\hline 1 & Providing evidence of the benefits and risks of DOACs in MPNs \\
\hline 2 & Providing evidence of the optimal duration of anticoagulant therapy in patients with MPN and thrombosis \\
\hline 3 & Increasing the evidence on efficacy of ruxolitinib as antithrombotic agents \\
\hline 4 & Improving knowledge of the association between driver mutations and risk factors for thrombosis in MPNs \\
\hline 5 & Testing the validity of surrogate endpoint for the experimental assessment of antithrombotic action of cytoreductive drugs in MPNs \\
\hline 6 & Providing evidence of the benefits and risks of cytoreduction in patients with SVT thrombosis without hypercythemia \\
\hline 7 & Providing evidence of the benefits and risks of JAK2 inhibitors in MPN-associated SVT \\
\hline 8 & Improving knowledge on risk factors of recurrence of thrombosis in MPNs \\
\hline 9 & Improving knowledge of the role acquired thrombophilia has on the development of thrombosis in MPNs \\
\hline 10 & Improving knowledge of the time from an MPN-associated thrombotic event and diagnosis of MPN \\
\hline 11 & Improving knowledge of the role of mutated endothelium in the pathogenesis of thrombosis in MPNs \\
\hline 12 & Improving knowledge of the leukocyte number as a risk factor for thrombosis and bleeding in MPNs \\
\hline 13 & Improving the diagnostic pathway of MPNs in the setting of splanchnic vein thrombosis \\
\hline 14 & Improving the diagnostic pathway of MPNs in the setting of cerebral vein thrombosis \\
\hline 15 & Optimizing the use of Willebrand test for assessing the risk of bleeding in extreme thrombocytosis in MPNs \\
\hline 16 & Improving the use of primary antithrombotic prophylaxis in patients with myelofibrosis \\
\hline 17 & Improving the dosing regiments of aspirin in ET \\
\hline 18 & Improving antithrombotic prophylaxis in MPN pregnant patients \\
\hline
\end{tabular}


In the cancer population, two recent prospective RCTs of edoxaban ${ }^{12}$ and rivaroxaban ${ }^{13}$, versus low-molecularweight heparin (LMWH) demonstrated the noninferiority of DOACs for VTE treatment in cancer patients.

In Ph-neg MPNs patients, VTE treatment with vitamin $\mathrm{K}$ antagonists (VKAs) provides good protection against recurrence, although rates of both recurrent thrombosis and major bleeding remain higher than in non-MPN patients ${ }^{14}$. DOACs can represent a suitable alternative, with the potential to protect both arterial and venous thrombosis and to perform better than VKAs in reducing bleeding risk $^{15}$. Knowledge of VTE treatment with DOACs in Ph-neg MPN patients is limited. In the two aforementioned trials, only $10 \%$ of recruited patients had hematological malignancies. In the OBENE registry, of the 760 MPN patients included, only 13 patients were receiving $\mathrm{DOACs}$ for $\mathrm{AF}$ and 12 for $\mathrm{VTE}^{16}$. In the German MPN registry of the Study Alliance Leukemia, 68 of 454 patients had deep vein thrombosis (DVT) or splanchnic vein thrombosis (SVT), and only eight were treated with rivaroxaban ${ }^{14}$.

The major issue in planning clinical trials with DOACs in cancer patients is trial feasibility. In the Hokusai trial ${ }^{12}$, a sample size of $\sim 1000$ patients was required, whereas in the SELECT-D trial, a target sample size of 530 was necessary ${ }^{13}$.

\section{Consensus statements}

Evidence of the benefits and risks of DOACs for the prevention of VTE recurrence has been so far achieved with noninferiority study designs in the normal population and in cancer patients. A noninferiority design is considered reasonable when evaluating new treatments that offer greater convenience for patients while providing similar efficacy.

It should be highlighted that the most conservative noninferiority margin assumes that the upper limit of the two-sided 95\% CIs of the hazard ratio is below the prespecified margin of 2.00. This margin sets the required sample size at approximately 1000 randomized patients.

In the setting of Ph-neg MPNs, which are rare diseases, the Panel agreed that a pragmatic pivotal randomized comparison of DOACs to the standard-treatment control, with the primary end-point defined as the recurrence of any VTE, could improve the trial's feasibility.

The investigators hypothesized that the benefit/harm profile of DOAC treatment will be noninferior to, or better than, usual care with LMWH/VKAs among the MPN patients. The information gained will empower MPN patients and physicians to make more informed choices about anticoagulation strategies to manage VTE.

Assuming a one-year VTE-free survival of 90\% (10\% cumulative incidence) for both arms, if there truly is no difference between the standard and experimental treatment, then 284 patients are required to be $80 \%$ sure that the upper limit of a one-sided $97.5 \%$ confidence interval will exclude a difference in favor of the standard group greater than 10\%. Allowing for 5\% nonevaluable patients, a total of 300 patients need to be randomized.

However, the Panel agreed that while awaiting the results of such a trial, the prevention of recurrent VTE in MPN patients should be based on VKA unless some individual factors prompt using DOACs.

UCN2: providing evidence of the benefits and risks of cytoreduction in patients with splanchnic vein thrombosis without hypercythemia

Cytoreductive therapy, along with full anticoagulation, represents the standard of care for patients with Ph-neg MPN and SVT ${ }^{17}$. However, a large proportion of patients do not exhibit classical phenotypic blood counts for $\mathrm{Ph}$ neg MPN at time of diagnosis (37.6 and $42.8 \%$ in recent multicenter cohort studies) ${ }^{18,19}$. The appropriateness of a myeloproliferation-targeted therapy in these patients is uncertain and has never been experimentally explored.

Approximately half of the JAK2V617F-positive SVT patients with a normal blood count at diagnosis will not develop an overt Ph-neg MPN during follow-up, and the clinical course of most of these patients is indolent ${ }^{20,21}$. Thus, a "watch-and-wait" approach, with cytoreduction starting only when a clear myeloproliferative pattern eventually appears, could be a reasonable approach.

The response to cytoreductive therapy in Ph-neg MPN patients with normal blood count is difficult to assess, since the current response criteria, developed for overt MPN, do not apply ${ }^{22,23}$. Two recently proposed SVT treatment algorithms in MPN do not recommend cytoreduction in patients with normal blood values ${ }^{17,24}$.

\section{Consensus statements}

The Panel agreed that the issue of benefits and risks of cytoreduction in patients with SVT without hypercythemia should be explored with an experimental approach.

The design of a randomized trial should address uncertainty in the simplest technical way, and the uncertainty principle should be used as the patient's eligibility criterion.

The target of therapy should be the reduction of hematological parameters down to the limit of threatening cytopenia.

The primary endpoint of the trial should be recurrence of thrombotic events.

However, the Panel highlighted the critical conundrum that the necessity of a randomized design conflicts with the necessity of collecting a large number of patients for testing a clinically relevant statistical hypothesis.

The Panel agreed that a prospective observational study should represent a feasible and informative solution that could be a first step forward toward preliminary 
information and toward creating a network of participating centers for a formal clinical trial.

UCN3: improving knowledge of the role of mutated endothelium in the pathogenesis of thrombosis in MPNs

Two independent reports have opened a new avenue for investigating the role of endothelial cells (ECs) in the pathogenesis of Ph-neg MPN-associated thrombosis. In 2009, Sozer et al. ${ }^{25}$ reported that the ECs of two patients with Budd-Chiari syndrome and PV were homozygous for $J A K 2$ V617F. In 2013, Rosti et al. ${ }^{26}$ documented that splenic ECs harbored the JAK2V617F mutation in patients with MF bearing the mutation in their granulocytes. Subsequently, the detection of either JAK2V617F- or CALR-mutated ECs in the bone marrow (BM) of patients with MF showed that the phenomenon is diffuse and not limited to the JAK2V617F mutation ${ }^{27}$.

Although the origin of the mutated endothelium is still under investigation ${ }^{28-30}$, its biological implications are relevant due to the functional consequences of the mutation at a cellular level ${ }^{31-35}$, and the phenomenon could be the dominant cause of endothelial dysfunction in $\mathrm{MPNs}^{36-41}$.

Investigation into circulating and tissue-derived ECs is technically challenging, and contradictory results are frequently reported ${ }^{42-47}$. In cultures from $\mathrm{BM}$, the main problem is overgrowth with pericytes or smooth muscle cells or contamination with fibroblasts, mesenchymal stromal cells and/or macrophages. However, it should be possible to set up cultures of ECs, free of non-EC contamination, by immunomagnetic depletion of monocyte/ macrophages, followed by positive selection of the remaining cells with beads coated with antibodies directed toward EC-specific antigens (VE-cadherin, CD31, CD146) and using EC-specific medium and fibronectin or collagen-coated flasks. This approach is hindered by the limited availability of $\mathrm{BM}$ aspirates, due to the fibrotic marrow of MF patients, and the lack of a standard single cell isolation technique from BM biopsies. The very low amount of circulating ECs discourages the use of peripheral blood as the source for EC isolation and purification ${ }^{48}$.

\section{Consensus statements}

The Panel agreed that the phenomenon of mutated endothelium in MPNs needs to move from a "proof of principle" issue to a study of a high number of wellrepresented patients.

For field advancement, there is a need for a direct, ex vivo approach to study the human endothelium in a condition as close as possible to its in vivo natural environment.

Endothelial cell purification and culture is proposed as the method of election. BM EC culture has the advantage of giving easy and ethically sustainable access to the relevant diseased organs in patients at risk of or with thrombotic complications.

The Panel claimed that optimization and standardization of EC cultures from BM-derived cells should be an impelling objective for the research community.

This technique should be used to answer relevant questions emerging after the discovery of a mutated endothelium in Ph-neg MPNs, i.e., its frequency, distribution, and its functional and clinical significance.

The association of a mutated endothelium with splanchnic vein thrombosis is a prime research topic that can be approached with cross-sectional analysis of patients with or without a thrombotic event (case-control study).

\section{UCN4: improving the dosing regimens of aspirin in ET}

No randomized trials have addressed the safety and efficacy of low-dose-aspirin (LDA) in ET such that, except for high-risk patients ${ }^{49-52}$, no definite data are available on its efficacy and safety, either alone or in association with hydroxyurea or anagrelide. The use of LDA in ET is justified based on indirect evidence and favorable results coming from the ECLAP study in PV $^{53}$. However, based on new prognostic scores in ET, observation alone is considered a viable option for "very low-risk" patients (aged $<60$ years, without history of thrombosis and/or risk factors, and unmutated $J A K 2 V 607 F$ ). In absence of contraindications, all other patients are thought to benefit from LDA therapy. However, this indication does not apply to secondary prophylaxis in which LDA is clearly indicated $^{54}$.

Single doses of $70-100 \mathrm{mg}$ nonenteric-coated aspirin are not questioned, since even $75 \mathrm{mg}$ is at least twice as high as the lowest dose necessary and sufficient to fully inhibit platelet COX-1 activity with repeated daily dosing $^{55}$. However, preclinical evidence supports more frequent dosing due to accelerated renewal of platelet COX1 secondary to the increased platelet production in ET, as indicated by the increased rate of immature platelets and by the measurement of serum TXB2 ${ }^{56,57}$. Measurement of this surrogate biomarker of aspirin efficacy showed that platelets from $80 \%$ of aspirin-treated ET patients were inadequately inhibited by the traditional, once-daily, lowdose regimen ${ }^{57}$. Hence, extrapolation to ET patients of the 100-mg once-daily regimen, borrowed from PV and cardiovascular disorders, does not seem to be justified in light of altered aspirin pharmacodynamics in $\mathrm{ET}^{55}$.

A twice-daily regimen of $100 \mathrm{mg}$ aspirin successfully improved the inhibition of serum $\mathrm{TXB}_{2}$ in a small, proofof-concept study ${ }^{58}$, and this schedule is already advised in high-risk patients ${ }^{59,60}$. On the other hand, twice-daily dosing may reduce compliance, inhibit prostacyclin $\left(\mathrm{PGI}_{2}\right)$ and increase bleeding. 


\section{Consensus statements}

The Panel agreed that optimization of LDA for primary prophylaxis of thrombosis in ET requires randomized trials investigating once daily versus more frequent administrations of LDA to evaluate clinical outcomes in terms of thrombosis and bleeding.

However, the Panel also argued that the most appropriate aspirin dose and dosing interval should be identified before conducting such a trial. This dosing information could be attained through a properly sized, dose-finding study that should measure mechanism-based, surrogate biomarkers of efficacy and safety.

Serum $T X B_{2}$ and urinary 2,3-dinor-6-keto-PGF ${ }_{1 a}{ }^{60}$ are the most attainable biomarkers to test the adequacy of platelet COX-1 inactivation and sparing of endothelial COX-2, respectively, throughout a variable dosing interval of aspirin in the low-dose range. Recently, the design of a prospective study to find the most appropriate schedule of LDA based on biological biomarker modifications has been published and results are awaited soon ${ }^{61}$.

\section{UCN5: improving antithrombotic management of Ph-neg} MPN-associated pregnancy

Management of pregnant women with Ph-neg MPNs is challenging because both pregnancy and MPN increase the risk of thrombosis, bleeding and obstetrical complications. In the absence of acquired von Willebrand disease or platelet dysfunction, antithrombotic prophylaxis with aspirin or/and LMWH during pregnancy and puerperium is considered along with cytoreduction with interferon (safe for the fetus) ${ }^{62,63}$.

No randomized or controlled study on efficacy or safety of antithrombotic or cytoreductive treatment in pregnant women with MPNs is available. Available data stem from case reports, small case series, and retrospective monocenter and multicenter cohorts often pooled in reviews ${ }^{64-74}$. One prospective observational study in pregnant women with MPNs is available ${ }^{75}$, but considering its several limitations, it does not add more solid data. However, the complication rates were lower than previously reported. Successful pregnancy is $96 \%$ versus $60-80 \%$, miscarriages are $1.7 \%$ versus $22-40 \%$, and thrombosis is absent. The rate of pregnancy-related thrombosis in cohort studies is $11-15 \%{ }^{76,77}$, but a metaanalysis reported an antepartum risk of $1.3 \%$ and $2.5 \%$ with and without LMWH, respectively, and a postpartum risk of $1.8 \%$ and $4.4 \%$ with and without LMWH, respectively $^{74}$. Thrombotic risk appears higher in women with PV than $\mathrm{ET}^{64-66}$. The risk of bleeding (mainly postpartum hemorrhage) varies between 3 and $12.5 \%^{74,76,77}$. The rate of placenta-mediated pregnancy complications appears higher than in the general population. Fetal growth restriction is $3-22 \%$, preeclampsia is $4-15 \%$, and stillbirth is $1.5-6 \%{ }^{78}$.
The role of the JAK2V167F mutation is controversial $^{66,79,80}$. A platelet count $>1000 \times 10^{9} / \mathrm{L}$, previous thrombosis, bleeding or obstetrical complications appear associated with an increased risk of obstetrical complications. Women with at least one of these predictors have a "high risk", and may deserve a more aggressive antithrombotic prophylaxis ${ }^{66,72}$. Apparently, age, parity, leukocyte or platelet count, and hemoglobin levels do not have predictive value ${ }^{66,75}$.

Data on the management of pregnant women with PMF are limited to fewer than 20 case reports ${ }^{81}$.

\section{Consensus statements}

The Panel agreed that evidence of the benefits and risks of more aggressive antithrombotic prophylaxis in pregnant women with Ph-neg MPN should ideally be acquired through a prospective, randomized, controlled trial with maternal thrombotic events, obstetrical complications, and bleeding events as a composite endpoint. However, the Panel highlighted that such a study with a randomized design conflicts with the need to collect the high number of patients needed to test a clinically relevant statistical hypothesis. Moreover, the Panel underlined the ethical reasons that prevent randomization of patients to potentially dangerous therapies without preliminary evidence of benefit.

Retrospective observational studies, such as those with a case-control design comparing patients who receive an aggressive antithrombotic prophylaxis or standard care have possible biases (indication, exclusion bias) that make the resulting evidence of limited quality.

The Panel agreed that a prospective observational study should be feasible and could provide preliminary information leading to creating a network of centers for a formal clinical trial with hypothesis testing as if women were to be randomized. Investigators will clearly state the purpose or main hypotheses, type of treatment and outcomes, possible confounders, primary analyses and sample size.

\section{UCN6: providing evidence of the optimal duration of anticoagulant therapy for prophylaxis of recurrent VTE}

The rate of recurrent VTE in the general population is $5-7 \%$ pt-years ${ }^{82}$. After discontinuation of anticoagulation, the recurrence rate at 1 and 5 years is $10 \%$ and $30 \%$ after unprovoked VTE, respectively, and 5\% and 15\% after VTE provoked by nonsurgical reversible factors, respectively $^{83,84}$. In non-MPN patients, these estimates drive the duration of VKA or DOAC treatment after VTE ranging from a minimum of 3 months to indefinite prolongation?

Several retrospective studies estimated the rate of recurrent thrombosis in Ph-neg MPN patients. The incidence of recurrent thrombosis was estimated to be $7.6 \%$ pt-years ${ }^{54}, 3.4$ on VKAs and 9.4 off VKAs, respectively $(p=0.016)^{85}$. In a single-center study, the recurrent VTE rate was $6.0 \%$ pt-years, with more events off $\mathrm{VKAs}^{86}$. In 
206 patients with well characterized diagnosis of DVT of the legs and/or PE, the incidence of recurrent thrombosis was $5.3 \%$ pt-years on VKAs and 12.8 after discontinuation $(p=0.008)$. After stopping VKAs, the recurrence rate was $42.3 \%$ at 5 years ${ }^{87}$. Patients with thrombosis of hepatic ${ }^{18}$ or cerebral veins ${ }^{88}$ were more prone to recurrences. Recurrent thrombosis can circumvent the effect of cytoreduction $^{54,85-87}$, and recent reappraisal of data from retrospective cohorts and clinical trials showed that the efficacy of hydroxyurea in preventing thrombosis is significant for arterial sites but doubtful for venous sites ${ }^{4,5}$.

A major concern during anticoagulation is bleeding risk. In non-MPN patients receiving VKAs, the rate of major bleeding is $1.2-2.2 \%^{89-92}$. In Ph-neg MPN patients, the incidence of major bleeding is $0.9-2.4 \%$ pt-years on VKAs and $0.7-1.5$ off VKAs and is as high as $2.8 \%$ pt-years when combining VKAs and aspirin ${ }^{54,85,87}$.

A recent survey reported high heterogeneity in treatment practices following thrombosis in Ph-neg patients ${ }^{93}$. Consensus statements and expert opinions suggested a prolongation of anticoagulation after unprovoked proximal DVT and/or PE, a life-threatening VTE or a VTE recurrence $^{94-96}$, but there are no controlled studies addressing this issue.

\section{Consensus statement}

The Panel agreed that further knowledge about the relationship between the duration of anticoagulation and the occurrence of rethrombosis in Ph-neg MPN patients is needed in order to provide evidence supporting decisions on anticoagulant prophylaxis of recurrent VTE.

This knowledge may allow the identification of categories of patients at different risk of rethrombosis and of bleeding, exploring the role played by potential risk factors (e.g., previous unprovoked VTE, proximal DVT, pulmonary embolism, male sex, D-dimer level, residual vein thrombosis, thrombophilia) or disease-related biomarkers (e.g. JAK2V617F mutation, leukocytosis).

To reach this goal, the Panel proposed a systematic review and meta-analysis of existing studies to summarize the evidence comparing various durations of secondary prophylaxis in Ph-neg MPNs. However, due to the literature being limited to cohort studies only, the pooled event rates and subgroup analyses will be the primary goal of such a meta-analysis.

The results could be the basis for a prospective observational study aimed to provide evidence for recommendations on duration of anticoagulant prophylaxis.

UCN7: improving the knowledge on the association between somatic mutations and risk factors for thrombosis in MPNs

An association of the JAK2V617F mutation and/or the variant allele frequency (VAF) with thrombosis was advocated in several retrospective analyses ${ }^{97-99}$. Patients with ET harboring the JAK2V617F mutation are at increased thrombotic risk, particularly if compared with CALR mutated or patients without known driver mutations ("triple negative") ${ }^{100,101}$; these findings led to the development of the IPSET score that includes JAK2V617F mutation as a risk variable (HR 2.0) ${ }^{3,102,103}$. Conversely, $J A K 2 \mathrm{~V} 617 \mathrm{~F}$ VAF is not currently included in risk scores for PV. Mechanistic explanations for the association of $J A K 2 \mathrm{~V} 617 \mathrm{~F}$ with thrombosis are scant and are mainly based on biological plausibility. Sustained activation of JAK2/STAT signaling causes erythrocytosis, leukocytosis and, to a lesser degree, thrombocytosis. Increased red cell mass is a major determinant of thrombotic events, as far known ${ }^{104}$ and supported by the CYTO-PV study ${ }^{105}$; although not prospectively validated yet, leukocytosis is associated with increased thrombosis rate ${ }^{106,107}$. Abnormal JAK2V617F-mediated Rap1-GTPase activation in neuthrophils, resulting in increased VCAM1/ICAM1mediated cell adhesion to the endothelium ${ }^{108}$ and neutrophil extracellular trap formation ${ }^{109}$, may mechanistically link mutated JAK2 to thrombosis pathogenesis. In addition, selected nondriver, myeloid neoplasmsassociated mutations (in particular, DNMT3A, TET2, $A S X L 1)$ may underlie an increased risk of thrombosis, since CHIP (Clonal Hematopoiesis of Indeterminate Potential), was associated with atherosclerotic cardiovascular disease in elderly healthy subjects ${ }^{110,111}$.

\section{Consensus statements}

The Panel agreed that the most straightforward approach of defining the value of driver mutations as predictive risk factors for thrombosis in Ph-neg MPN should be a prospective study.

Although thrombosis may occur at a similar rate in PMF and ET, the complexity of clinical course and the confounding effects of therapies make PMF unsuitable for investigation; therefore, in principle, studies should be limited to patients with PV and ET.

One should accept the intrinsic variabilities of a "reallife" approach and perform prospective observational studies that include patients enrolled and genotyped at the time of diagnosis and followed according to the "bestpractice", until a predefined number of events is registered. An extensive database with all potential variables currently known to influence thrombosis rate should be used for multivariable analysis; accordingly, the number of patients to include may be indeed very high.

\section{UCN8: improving the grading system of the risk of thrombosis in PV}

Current risk stratification in PV is designed to estimate the likelihood of future arterial and venous thrombosis and considers low- and high-risk categories based on the 
respective absence or presence of either age $>60$ years or history of thrombosis. A matter of discussion is whether these definitions predicting thrombotic risk are still valid and whether the newly proposed disease-related risk factors, such as cardiovascular risk factors, leukocytosis, JAK2V617F allele burden, may improve the grading system of the thrombotic risk.

The Panel claimed that white blood cell (WBC) represents a clinically relevant candidate risk factor. As a matter of fact, the prognostic role of elevated WBC counts, but not thrombocytosis, was demonstrated in subanalysis of randomized clinical trials and in multicenter observational studies ${ }^{63}$. In a subanalysis of the ECLAP trial ${ }^{106}$, WBC $>17 \times 10^{9} / \mathrm{L}$ was independently associated with myocardial infarction, and in patients of the high-hematocrit arm of CYTO-PV trial, the risk of thrombosis was clearly increased in the presence of WBC count above $6 \times 10^{9} / \mathrm{L}$, becoming statistically significant when WBC count was above $12 \times 10^{9} / \mathrm{L}$ (hazard ratio, 4.89; 95\% confidence interval $(\mathrm{CI}), 1.1-22.7 ; P=0.04)^{112}$. Whether leukocytosis is a marker or a causative factor for thrombosis is a matter of discussion ${ }^{113}$.

\section{Consensus statements}

The Panel agreed that the major challenge in using leukocyte count as a risk factor for thrombosis in PV is the acquisition of strong evidence that a threshold value for leucocyte count represents a risk factor for thrombosis.

With such knowledge, experimental studies could be planned to evaluate thrombotic risks and benefits in terms of thrombotic event reduction with cytoreductive therapy.

The Panel proposed a comprehensive systematic review and meta-analysis of existing observational studies as the best method for summarizing the evidence comparing various event rates at different leukocyte counts.

The quality of the studies (good, fair and poor) should be assessed by awarding scores in each domain using a validated methodological evaluation tool, such as the Newcastle-Ottawa Scale (NOS), and publication bias should be properly evaluated.

The aims of this systematic review and meta-analysis should be to assess leukocyte count relative risk estimates associated with arterial and venous major thrombotic events in adult patients with $P V$, to verify the results of the meta-analysis in subgroups of patients with ET and $P V$, and to evaluate a possible WBC cut-off able to predict vascular complications.

The Panel agreed that the results of the meta-analysis could provide a basis for testing the prognostic accuracy of the best cut-off of WBC count in large, existing international registries, which could subsequently be incorporated into the grading system of thrombotic risk in PV and ET.

\section{Conclusion}

In this paper, UCNs with greater clinical relevance for Ph-neg MPN-associated thrombosis were identified, and consensus-based statements were issued by a Panel of experts and addressed mainly to the scientific community. Even though the molecular revolution that followed the discovery of the JAK2V617F mutation has opened several new research paths, of the eight selected UCNs, three addressed the diagnosis or risk stratification of thrombotic events. This decision argues that standardization of diagnostic pathways and patient risk stratification are prerequisites for any research on new therapeutics.

The major discussions about the proposed solutions for the UCNs were of methodological grade. As a matter of fact, many needs for Ph-neg MPN-associated thrombosis resulted from practical difficulties in increasing experimental knowledge in the field. This need mostly derives from the issue of the rarity of Ph-neg MPN patients, which urges designing nonrandomized studies with the power to provide new strong experimental knowledge. The Panel largely drew in a high number of methodological novelties proposed to solve the rarity problem, particularly with regard to the risk of selection and motivation biases ${ }^{114-116}$.

The hope is that this comprehensive overview will serve to inform the design and implementation of research in the field and will help the management of special clinical situations in these disorders.

\section{Acknowledgements}

The authors wish to thank the ELN European guests participating in the meeting for their valuable contributions: Alberto Alvarez-Larran (Barcelona, ES), Gunnar Birgegård (Uppsala, SE), Francisco Cervantes (Barcelona, ES), Heinz Gisslinger (Wien, A), Martin Griesshammer (Minden, DE), Rüdiger Hehlmann (Mannheim, DE), Juan Carlos Hernández Boluda (Valencia, ES), Chloé James (Bordeaux, FR), Jean-Jacques Kiladjian (Paris, FR), Steffen Koschmieder (Aachen, DE), Mary Frances McMullin (Belfast, UK), Francesco Passamonti (Varese, IT), Johanne Poisson (Paris, FR), Andreas Reiter (Mannheim, DE), Susan Robinson (London, UK), and Bianca Rocca (Rome, IT). Special thanks to Menarini International Foundation (Florence, IT) for supporting this study and the Workshop held in Bergamo (IT) in January 2019.

\section{Author details}

${ }^{1}$ FROM Research Foundation, Papa Giovanni XXIII Hospital, Bergamo, Italy. ${ }^{2}$ Fondazione Policlinico Universitario A. Gemelli IRCCS, Roma, Italy. ${ }^{3}$ Istituto di Ematologia, Università Cattolica, Roma, Italy. ${ }^{4}$ Department of Immunohematology and Transfusion Medicine and the Haemostasis and Thrombosis Center, Papa Giovanni XXIII Hospital, Bergamo, Italy. ${ }^{5}$ University of Milan Bicocca, Milan, Italy. ${ }^{6}$ Hematology Division, Papa Giovanni XXIII Hospital, Bergamo, Italy. ${ }^{7}$ Fondazione IRCCS Ca' Granda Ospedale Maggiore Policlinico, A. Bianchi Bonomi Hemophilia and Thrombosis Center, Milan, Italy.

${ }^{8}$ Hematology Project Foundation, affiliated to the Department of Hematology, San Bortolo Hospital, Vicenza, Italy. ${ }^{9}$ CRIMM-Center of Research and Innovation of Myeloproliferative Neoplasms, Azienda Ospedaliera Universitaria Careggi, Dept Experimental and Clinical medicine, and Denothe Center, University of Florence, Florence, Italy. ${ }^{10}$ Center for the Study of Myelofibrosis, IRCCS

Policlinico S. Matteo Foundation, Pavia, Italy 


\section{Conflict of interest}

The authors declare that they have no conflict of interest.

\section{Publisher's note}

Springer Nature remains neutral with regard to jurisdictional claims in published maps and institutional affiliations.

Received: 29 March 2019 Revised: 6 May 2019 Accepted: 14 May 2019 Published online: 08 August 2019

\section{References}

1. Rampal, R. \& Levine, R. L. A primer on genomic and epigenomic alterations in the myeloproliferative neoplasms. Best Pract. Res. Clin. Haematol. 27, 83-93 (2014).

2. Barbui, T., Finazzi, G. \& Falanga, A. Myeloproliferative neoplasms and thrombosis. Blood 122, 2176-2084 (2013).

3. Barbui, T. et al. Development and validation of an International Prognostic Score of thrombosis in World Health Organization-essential thrombocythemia (IPSET-thrombosis). Blood 120, 5128-5133 (2012).

4. Barbui, T. et al. Different effect of hydroxyurea and phlebotomy on prevention of arterial and venous thrombosis in polycythemia vera. Blood Cancer J. 8, 124 (2018).

5. De Stefano, V. et al. Hydroxyurea prevents arterial and late venous thrombotic recurrences in patients with myeloproliferative neoplasms but fails in the splanchnic venous district. Pooled analysis of 1500 cases. Blood Cancer J. 8, 112 (2018).

6. Hultcrantz, M. et al. Risk for arterial and venous thrombosis in patients with myeloproliferative neoplasms: a population-based cohort study. Ann. Intern. Med. 168, 317-325 (2018).

7. Rungjirajittranon, T., Owattanapanich, W., Ungprasert, P., Siritanaratkul, N. \& Ruchutrakool, T. A systematic review and meta-analysis of the prevalence of thrombosis and bleeding at diagnosis of Philadelphia-negative myeloproliferative neoplasms. BMC Cancer 19, 184 (2019).

8. Kaifie, A. et al. Health care setting and severity, symptom burden, and complications in patients with Philadelphia-negative myeloproliferative neoplasms (MPN): a comparison between university hospitals, community hospitals, and office-based physicians. Ann. Hematol. 95, 1399-1410 (2016).

9. Delbecq, A. L., Van de Ven, A. H. \& Gustafson, D. H. Group Techniques for Program Planning: A guide to nominal group and Delphi processes. (Scott, Foresman and Co, Glenview, IL, 1975).

10. Kearon, C. et al. Antithrombotic therapy for VTE disease: CHEST guideline and expert panel report. Chest 149, 315-352 (2016).

11. Steffel, J. et al. The 2018 European Heart Rhythm Association practical guide on the use of non-vitamin $\mathrm{K}$ antagonist oral anticoagulants in patients with atrial fibrillation. Eur. Heart J. 39, 1330-1393 (2018).

12. Raskob, G. E. et al. Hokusai VTE cancer investigators. Edoxaban for the treatment of cancer-associated venous thromboembolism. N. Engl. J. Med. 378, 615-624 (2018).

13. Young, A. M. et al. Comparison of an oral factor Xa inhibitor with low molecular weight heparin in patients with cancer with venous thromboembolism: results of a randomized trial (SELECT-D). J. Clin. Oncol. 36, 2017-2023 (2018).

14. Kaifie, A. et al. Study Alliance Leukemia (SAL). Bleeding, thrombosis, and anticoagulation in myeloproliferative neoplasms (MPN): analysis from the German SAL-MPN-registry. J. Hematol. Oncol. 9, 18 (2016),

15. Gómez-Outes, A. et al. Case fatality rates of recurrent thromboembolism and bleeding in patients receiving direct oral anticoagulants for the initial and extended treatment of venous thromboembolism: a systematic review. J. Cardiovasc. Pharmacol. Ther. 20, 490-500 (2015).

16. lanotto, J. C. et al. Administration of direct oral anticoagulants in patients with myeloproliferative neoplasms. Int. J. Hematol. 106, 517-521 (2017).

17. Finazzi, G., De Stefano, V. \& Barbui, T. Splanchnic vein thrombosis in myeloproliferative neoplasms: treatment algorithm 2018. Blood. Cancer J. 8, 64 (2018).

18. De Stefano, V. et al. Splanchnic vein thrombosis in myeloproliferative neoplasms: risk factors for recurrences in a cohort of 181 patients. Blood Cancer J. 6, e493 (2016)
19. Greenfield, G. \& McMullin, M. F. Splanchnic venous thrombosis in JAK2 V617F mutation positive myeloproliferative neoplasms-long term follow-up of a regional case series. Thromb. J. 16, 33 (2018).

20. Colaizzo, D. et al. Outcome of patients with splanchnic venous thrombosis presenting without overt MPN: a role for the JAK2 V617F mutation reevaluation. Thromb. Res. 132, e99-e104 (2013)

21. Dentali, F. et al. JAK2V617F mutation for the early diagnosis of Ph- myeloproliferative neoplasms in patients with venous thromboembolism: a metaanalysis. Blood 113, 5617-5623 (2009).

22. Tefferi, A. et al. Revised response criteria for myelofibrosis: International Working Group-Myeloproliferative Neoplasms Research and Treatment (IWGMRT) and European LeukemiaNet (ELN) consensus report. Blood 122 1395-1398 (2013).

23. Barosi, G. et al. Revised response criteria for polycythemia vera and essential thrombocythemia: an ELN and IWG-MRT consensus project. Blood 121, 4778-4781 (2013).

24. De Stefano, V., Qi, X., Betti, S. \& Rossi, E. Splanchnic vein thrombosis and myeloproliferative neoplasms: molecular-driven diagnosis and long-term treatment. Thromb. Haemost 115, 240-249 (2016).

25. Sozer, S. et al. The presence of JAK2V617F mutation in the liver endothelial cells of patients with Budd-Chiari syndrome. Blood 113, 5246-5249 (2009).

26. Rosti, V. et al. Spleen endothelial cells from patients with myelofibrosis harbor the JAK2V617F mutation. Blood 121, 360-368 (2013).

27. Villani, L. et al. V617FJAK2-positive endothelial cells are present in bone marrow neovessels of patients with myelofibrosis and could derive from the transdifferentiation of mutated hematopoietic cells. Blood 126 (57th Congress of the American Society of Hematology, Orlando (FL), 2015). Meeting abstract: 2833 (poster)December 5-8.

28. Teofili, L. et al. Endothelial progenitor cells are clonal and exhibit the JAK2 (V617F) mutation in a subset of thrombotic patients with Ph-negative myeloproliferative neoplasms. Blood 117, 2700-2707 (2011).

29. Piaggio, G. et al. Endothelial colony-forming cells from patients with chronic myeloproliferative disorders lack the disease-specific molecular clonality marker. Blood 114, 3127-3130 (2009).

30. Sozer, S. et al. Human CD34+ cells are capable of generating normal and JAK2V617F positive endothelial like cells in vivo. Blood Cells Mol. Dis. 43, 304-312 (2009).

31. Anand, S. \& Cheresh, D. A. MicroRNA-mediated regulation of the angiogenic switch. Curr. Opin. Hematol. 18, 171-176 (2011).

32. Bartoli, M. et al. VEGF differentially activates STAT3 in microvascular endothelial cell S. FASEB J. 17, 1562-1564 (2003).

33. Betel, D., Wilson, M., Gabow, A., Marks, D. S. \& Sander, C. The microRNA.org resource: targets and expression. Nucleic Acids Res. 36, D149-D153 (2008).

34. Dong, $Y$. et al. Cucurbitacin $E$, a tetracyclic triterpenes compound from Chinese medicine, inhibits tumor angiogenesis through VEGFR2-mediated Jak2-STAT3 signaling pathway. Carcinogenesis 31, 2097-2104 (2010).

35. Leong, H., Mathur, P. S. \& Greene, G. L. Green tea catechins inhibit angiogenesis through suppression of STAT3 activation. Breast Cancer Res. Treat 117, 505-515 (2009).

36. Yildiz, A. et al. Assessment of endothelial dysfunction with flow-mediated dilatation in myeloproliferative disorders. Clin. Appl. Thromb. Hemost. 24, 1102-1108 (2018).

37. Neunteufl, T., Heher, S., Stefenelli, T., Pabinger, I. \& Gisslinger, H. Endothelial dysfunction in patients with polycythaemia vera. Br. J. Haematol. 115, 354-359 (2001).

38. Etheridge, S. L. et al. JAK2V617F-positive endothelial cells contribute to clotting abnormalities in myeloproliferative neoplasms. Proc. Natl Acad. Sci. USA 111, 2295-2300 (2014).

39. Guadall, A. et al. Endothelial cells harbouring the JAK2V617F mutation display pro-adherent and pro-thrombotic features. Thromb. Haemost. 118, 1586-1599 (2018).

40. Guy, A. et al. Vascular endothelial cell expression of JAK2V617F is sufficient to promote a pro-thrombotic state due to increased P-selectin expression. Haematologica 104, 70-81 (2019).

41. Lin, C. H., Kaushansky, K. \& Zhan, H. JAK2V617F-mutant vascular niche contributes to JAK2V617F clonal expansion in myeloproliferative neoplasms. Blood Cells Mol. Dis. 62, 42-48 (2016).

42. Boraldi, F. et al. Innovative flow cytometry allows accurate identification of rare circulating cells involved in endothelial dysfunction. PLOS ONE 11, e0160153 (2016) 
43. Rosso, V. et al. A novel assay to detect calreticulin mutations in myeloproliferative neoplasms. Oncotarget 8, 6399-6405 (2017).

44. Sozer, S. \& Hoffman, R. Laser-capture microdissection and analysis of liver endothelial cells from patients with Budd-Chiari syndrome. Methods Mol. Biol. 755, 405-415 (2011).

45. Rafii, S. et al. Human bone marrow microvascular endothelial cells support long-term proliferation and differentiation of myeloid and megakaryocytic progenitors. Blood 86, 3353-3363 (1995).

46. Wang, Q. R., Yan, Y., Wang, B. H., Li, W. M. \& Wolf, N. S. Long-term culture of murine bone-marrow-derived endothelial cells. In Vitro Cell. Dev. Biol. Anim. 34, 443-446 (1998).

47. Vacca, A. et al. Endothelial cells in the bone marrow of patients with multiple myeloma. Blood 102, 3340-3348 (2003).

48. Lanuti, P. et al. A standardized flow cytometry network study for the assessment of circulating endothelial cell physiological ranges. Sci. Rep. 8 5823 (2018).

49. Patrono, C., Rocca, B. \& De Stefano, V. Platelet activation and inhibition in polycythemia vera and essential thrombocythemia. Blood 121, 1701-1711 (2013).

50. van Genderen, P. J., Mulder, P. G., Waleboer, M., van de Moesdijk, D. \& Michiels, J. J. Prevention and treatment of thrombotic complications in essential thrombocythaemia: efficacy and safety of aspirin. Br. J. Haematol. 97, 179-184 (1997).

51. Alvarez-Larran, A. et al. Observation versus antiplatelet therapy as primary prophylaxis for thrombosis in low-risk essential thrombocythemia. Blood 116, 1205-1210 (2010).

52. Alvarez-Larran, A. et al. Cytoreduction plus low-dose aspirin versus cytoreduction alone as primary prophylaxis of thrombosis in patients with highrisk essential thrombocythaemia: an obervational study. Br. J. Haematolol. 161, 865-871 (2013).

53. Landolfi, R. et al. Efficacy and safety of low-dose aspirin in polycythemia vera. N. Engl. J. Med. 350, 114-124 (2004)

54. De Stefano, V. et al. Recurrent thrombosis in patients with polycythemia vera and essential thrombocythemia: incidence, risk factors, and effect of treatments. Haematologica 93, 372-380 (2008).

55. Patrignani, P., Filabozzi, P. \& Patrono, C. Selective cumulative inhibition of platelet thromboxane production by low-dose aspirin in healthy subjects. J. Clin. Investig. 69, 1366-1372 (1982).

56. Panova-Noeva, M. et al. JAK2V617F mutation and hydroxyurea treatment as determinants of immature platelet parameters in essential thrombocythemia and polycythemia vera patients. Blood 118, 2599-2601 (2011).

57. Dragani, A. et al. The contribution of cyclooxygenase-1 and -2 to persistent thromboxane biosynthesis in aspirin-treated essential thrombocythemia: implications for antiplatelet therapy. Blood 115, 1054-1061 (2010).

58. Pascale, S. et al. Aspirin-insensitive thromboxane biosynthesis in essential thrombocythemia is explained by accelerated renewal of the drug target. Blood 119, 3595-3603 (2012).

59. Tefferi, A. \& Barbui, T. Personalized management of essential thrombocythemia-application of recent evidence to clinical practice. Leukemia 27, 1617-1620 (2013).

60. FitzGerald, G. A. et al. Endogenous biosynthesis of prostacyclin and thromboxane and platelet function during chronic administration of aspirin in man. J. Clin. Invest. 71, 676-688 (1983).

61. De Stefano, V. et al. The Aspirin Regimens in Essential Thrombocythemia (ARES) phase II randomized trial design: Implementation of the serum thromboxane B2 assay as an evaluation tool of different aspirin dosing regimens in the clinical setting. Blood Cancer J. 8, 49 (2018).

62. Barbui, T. et al. Philadelphia-negative classical myeloproliferative neoplasms: critical concepts and management recommendations from European leukemiaNet. J. Clin. Oncol. 29, 761-770 (2011).

63. Tefferi, A., Vannucchi, A. M. \& Barbui, T. Polycythemia vera treatment algorithm 2018. Blood Cancer J. 8, 3 (2018).

64. Griesshammer, M., Struve, S. \& Harrison, C. M. Essential thrombocythemia/ polycythemia vera and pregnancy: the need for an observational study in Europe. Semin. Thromb. Hemost. 32, 422-429 (2006).

65. Barbui T., Finazzi G. Myeloproliferative disease in pregnancy and other management issues. Hematol. Am. Soc. Hematol. Educ. Prog. 246-252 (2006)

66. Griesshammer, M., Struve, S. \& Barbui, T. Management of Philadelphia negative chronic myeloproliferative disorders in pregnancy. Blood Rev. 22 235-245 (2008).
67. Harrison, C. N. \& Robinson, S. E. Myeloproliferative disorders in pregnancy. Hematol. Oncol. Clin. North Am. 25, 261-275 (2011).

68. Valera, M. C., Parant, O., Vayssiere, C., Arnal, J. F. \& Payrastre, B. Essential thrombocythemia and pregnancy. Eur. J. Obstet. Gynecol. Reprod. Biol. 158, 141-147 (2011).

69. Kreher, S. et al. Prophylaxis and management of venous thromboembolism in patients with myeloproliferative neoplasms: consensus statement of the Haemostasis Working Party of the German Society of Hematology and Oncology (DGHO), the Austrian Society of Hematology and Oncology (ÖGHO) and Society of Thrombosis and Haemostasis Research (GTH e.V.). Ann. Hematol. 93, 1953-1963 (2014).

70. Kim, J., Haddad, R. Y. \& Atallah, E. Myeloproliferative neoplasms. Dis. Mon. 58, 177-194 (2012).

71. Kiladjian J. J. Current therapies and their indications for the Philadelphianegative myeloproliferative neoplasms. Am. Soc. Clin. Oncol. Educ. Book. e389-e396 (2015).

72. Horowitz, N. A., Lavi, N., Nadir, Y. \& Brenner, B. Haematological malignancies in pregnancy: an overview with an emphasis on thrombotic risks. Thromb. Haemost. 116, 613-617 (2016).

73. Mahmoud, H. K., Samra, M. A. \& Fathy, G. M. Hematologic malignancies during pregnancy: a review. J. Adv. Res. 7, 589-596 (2016).

74. Skeith, L., Carrier, M., Robinson, S. E., Alimam, S. \& Rodger, M. A. Risk of venous thromboembolism in pregnant women with essential thrombocythemia: A systematic review and meta-analysis. Blood 129, 934-939 (2017).

75. Alimam, S. et al. Pregnancy outcomes in myeloproliferative neoplasms: UK prospective cohort study. Br. J. Haematol. 175, 31-36 (2016)

76. Randi, M. L. et al. Pregnancy complications predict thrombotic events in young women with essential thrombocythemia. Am. J. Hematol. 89, 306-309 (2014).

77. Lapoirie J. et al. Management and outcomes of 27 pregnancies in women with myeloproliferative neoplasms. J. Matern. Fetal Neonatal Med. 1-8 (2018)

78. Umazume, T. et al. Essential thrombocythemia as a risk factor for stillbirth. Thromb. Res. 133, 158-161 (2014).

79. Passamonti, F., Rumi, E., Randi, M. L., Morra, E. \& Cazzola, M. Aspirin in pregnant patients with essential thrombocythemia: a retrospective analysis of 129 pregnancies. J. Thromb. Haemost. 8, 411-413 (2010).

80. Palandri, F. et al. Long-term follow-up of essential thrombocythemia in young adults: treatment strategies, major thrombotic complications and pregnancy outcomes. A study of 76 patients. Haematologica 95, 1038-1040 (2010).

81. Griesshammer, M., Sadjadian, P. \& Wille, K. Contemporary management of patients with BCR-ABL1-negative myeloproliferative neoplasms during pregnancy. Expert Rev. Hematol. 11, 697-706 (2018).

82. Cushman, M. Epidemiology and risk factors for venous thrombosis. Semin. Hematol. 44, 62-69 (2007).

83. Kearon, C., lorio, A. \& Palareti, G. Subcommittee on control of anticoagulation of the SSC of the ISTH. Risk of recurrent venous thromboembolism after stopping treatment in cohort studies: recommendation for acceptable rates and standardized reporting. J. Thromb. Haemost. 8, 2313-2315 (2010).

84. Baglin, T. et al. Duration of anticoagulant therapy after a first episode of an unprovoked pulmonary embolus or deep vein thrombosis: guidance from the SSC of the ISTH. J. Thromb. Haemost. 10, 698-702 (2012).

85. Hernández-Boluda, J. C. et al. Grupo Español de Enfermedades Mieloproliferativas Filadelfia Negativas (GEMFIN). Oral anticoagulation to prevent thrombosis recurrence in polycythemia vera and essential thrombocythemia. Ann. Hematol. 94, 911-918 (2015).

86. Wille, K. et al. High risk of recurrent venous thromboembolism in BCR-ABLnegative myeloproliferative neoplasms after termination of anticoagulation. Ann. Hematol. 98, 93-100 (2019).

87. De Stefano, V. et al. High rate of recurrent venous thromboembolism in patients with myeloproliferative neoplasms and effect of prophylaxis with vitamin K antagonists. Leukemia 30, 2032-2038 (2016).

88. Martinelli, I. et al. Cerebral vein thrombosis in patients with Philadelphianegative myeloproliferative neoplasms. An European Leukemia Net study. Am. J. Hematol. 89, E200-E205 (2014).

89. Bauersachs, R. et al. Oral rivaroxaban for symptomatic venous thromboembolism. N. Engl. J. Med. 363, 2499-2510 (2010).

90. Büller, H. R. et al. Oral rivaroxaban for the treatment of symptomatic pulmonary embolism. N. Engl. J. Med. 366, 1287-1297 (2012).

91. Agnelli, G. et al. Oral apixaban for the treatment of acute venous thromboembolism. N. Engl. J. Med. 369, 799-808 (2013). 
92. Buller, H. R. et al. Edoxaban versus warfarin for the treatment of symptomatic venous thromboembolism. N. Engl. J. Med. 369, 1406-1415 (2013).

93. Ellis, M. H., Lavi, N., Vannucchi, A. \& Harrison, C. Treatment of thromboembolic events coincident with the diagnosis of myeloproliferative neoplasms: a physician survey. Thromb. Res. 134, 251-254 (2014).

94. Kreher, S. et al. Prophylaxis and management of venous thromboembolism in patients with myeloproliferative neoplasm: consensus statement of DGHO, ÖGHO and GTH. Ann. Hematol. 93, 1953-1963 (2014).

95. Tefferi, A. \& Barbui, T. Polycythemia vera and essential thrombocythemia: 2017 update on diagnosis, risk-stratification and management. Am. J. Hematol. 92, 94-108 (2017).

96. De Stefano, V., Finazzi, G. \& Barbui, T. Antithrombotic therapy for venous thromboembolism in myeloproliferative neoplasms. Blood Cancer J. 8, 65 (2018).

97. Vannucchi, A. M. et al. Clinical profile of homozygous JAK2V617F mutation in patients with polycythemia vera or essential thrombocythemia. Blood $\mathbf{1 1 0}$ 840-846 (2007).

98. Vannucchi, A. M., Antonioli, E., Guglielmelli, P., Pardanani, A. \& Tefferi, A. Clinical correlates of JAK2V617F presence or allele burden in myeloproliferative neoplasms: a critical reappraisal. Leukemia 22, 1299-1307 (2008).

99. Vannucchi, A. M. et al. Prospective identification of high-risk polycythemia vera patients based on JAK2V617F allele burden. Leukemia 21, 1952-1959 (2007).

100. Rotunno, G. et al. Impact of calreticulin mutations on clinical and hematological phenotype and outcome in essential thrombocythemia. Blood 123, 1552-1555 (2014).

101. Rumi, E. et al. JAK2 or CALR mutation status defines subtypes of essential thrombocythemia with substantially different clinical course and outcomes. Blood 123, 1544-1551 (2014).

102. Barbui, T. et al. Practice-relevant revision of IPSET-thrombosis based on 1019 patients with WHO-defined essential thrombocythemia. Blood Cancer J. 5, e369 (2015)
103. Finazzi, G. et al. Calreticulin mutation does not modify the IPSET score for predicting the risk of thrombosis among 1150 patients with essential thrombocythemia. Blood 124, 2611-2612 (2014).

104. Pearson, T. C. \& Wetherley-Mein, G. Vascular occlusive episodes and venous haematocrit in primary proliferative polycythaemia. Lancet 2, 1219-1222 (1978).

105. Marchioli, R. et al. Cardiovascular events and intensity of treatment in polycythemia vera. N. Engl. J. Med. 368, 22-33 (2013).

106. Landolfi, R. et al. Leukocytosis as a major thrombotic risk factor in patients with polycythemia vera. Blood 109, 2446-2452 (2007).

107. Carobbio, A. et al. Leukocytosis and risk stratification assessment in essential thrombocythemia. J. Clin. Oncol. 26, 2732-2736 (2008).

108. Edelmann, B. et al. JAK2-V617F promotes venous thrombosis through beta1/ beta2 integrin activation. J. Clin. Investig. 128, 4359-4371 (2018).

109. Wolach, O. et al. Increased neutrophil extracellular trap formation promotes thrombosis in myeloproliferative neoplasms. Sci. Transl. Med. 10, eaan8292 (2018).

110. Jaiswal, S. et al. Age-related clonal hematopoiesis associated with adverse outcomes. N. Engl. J. Med. 371, 2488-2498 (2014).

111. Jaiswal, S. et al. Clonal hematopoiesis and risk of atherosclerotic cardiovascular disease. N. Engl. J. Med. 377, 111-121 (2017).

112. Barbui, T. et al. White blood cell counts and thrombosis in polycythemia vera: a subanalysis of the CYTO-PV study. Blood 126, 560-561 (2015).

113. Barbui, T. Carobbio, A., Rambaldi, A. \& Finazzi, G. Perspectives on thrombosis in essential thrombocythemia and polycythemia vera: is leukocytosis a causative factor? Blood 114, 759-763 (2009).

114. Moore, C. G., Carter, R. E., Nietert, P. J. \& Stewart, P. W. Recommendations for planning pilot studies in clinical and translational research. Clin. Transl. Sci. 4, 332-337 (2011).

115. Rubinstein, L. V. et al. Design issues of randomized phase ॥ trials and a proposal for phase II screening trials. J. Clin. Oncol. 23, 7199-7206 (2005).

116. Berger, M. L. et al. Prospective observational studies to assess comparative effectiveness: the ISPOR good research practices task force report. Value Health 15, 217-230 (2012). 